\title{
SENSITIVITY ANALYSIS OF ORDERED WEIGHTED AVERAGING OPERATOR IN EARTHQUAKE VULNERABILITY ASSESSMENT
}

\author{
M. Moradi ${ }^{\text {a }}$, M.R. Delavar ${ }^{\mathrm{b}}$ and B. Moshiri ${ }^{\mathrm{c}}$ \\ ${ }^{a}$ Dept. of Surveying and Geomatics Eng., University of Tehran, Tehran, Iran- (milad.moradi, fa_khamespanah, \\ abbas.zoghian)@ut.ac.ir \\ ${ }^{\mathrm{b}}$ Center of Excellence in Geomatics Eng. in Disaster Management, Dept. of Surveying and Geomatics Eng., College of Eng., \\ University of Tehran, Tehran, Iran-mdelavar@ut.ac.ir \\ ${ }^{\mathrm{c}}$ Control and Intelligent Processing Center of Excellence, School of Electrical and Computer Eng., University of Tehran, Tehran, \\ Iran-moshiri@ut.ac.ir
}

KEY WORDS: Sensitivity Analysis, Ordered Weighted Average, Earthquake Vulnerability Assessment, Minimal Variability

\begin{abstract}
:
The main objective of this research is to find the extent to which the minimal variability Ordered Weighted Averaging (OWA) model of seismic vulnerability assessment is sensitive to variation of optimism degree. There are a variety of models proposed for seismic vulnerability assessment. In order to examine the efficiency of seismic vulnerability assessment models, the stability of results could be analysed. Seismic vulnerability assessment is done to estimate the probable losses in the future earthquake. Multi-Criteria Decision Making (MCDM) methods have been applied by a number of researchers to estimate the human, physical and financial losses in urban areas. The study area of this research is Tehran Metropolitan Area (TMA) which has more than eight million inhabitants. In addition, this paper assumes that North Tehran Fault (NTF) is activated and caused an earthquake in TMA. 1996 census data is used to extract the attribute values for six effective criteria in seismic vulnerability assessment. The results demonstrate that minimal variability OWA model of Seismic Loss Estimation (SLE) is more stable where the aggregated seismic vulnerability degree has a lower value. Moreover, minimal variability OWA is very sensitive to optimism degree in northern areas of Tehran. A number of statistical units in southern areas of the city also indicate considerable sensitivity to optimism degree due to numerous non-standard buildings. In addition, the change of seismic vulnerability degree caused by variation of optimism degree does not exceed $25 \%$ of the original value which means that the overall accuracy of the model is acceptable.
\end{abstract}

\section{INTRODUCTION}

Natural disasters have long been one of the main sources of damage to human societies (Crowley and Bommer, 2006). Earthquakes are known as one of the most destructive disasters which can cause a huge damage in very few seconds. Tehran, capital of Iran, is threatened with a number of known and unknown faults surrounding the city (Alinia and Delavar, 2011). In addition, high population, rapid expansion and non-standard constructions are turning Tehran into an extremely vulnerable city to earthquake ( Samadi Alinia and Delavar, 2011; Hashemi and Alesheikh, 2012; Khamespanah et al., 2013). Extensive researches has been undertaken to estimate the loss of property and human life in the future earthquake of Tehran . A number of researches proposed seismic loss estimation models based on multi-criteria decision making algorithms (Kolat et al., 2006; Mohan et al., 2007; Rashed and Weeks, 2003). The main framework of these models include: (1) determining the contributing criteria that affect the seismic vulnerability of an urban area (2) extracting and normalizing the attribute values (3) specifying the experts' opinion (4) aggregating the attribute values using MCDM algorithms and calculating the vulnerability degrees. Therefore, seismic vulnerability models do not have the same results necessarily. On the other hand, Tehran has not experienced any earthquake since 1830 (Hashemi and Alesheikh, 2012). Consequently, independent validation of Tehran's seismic loss estimation models is impossible due to lack of real data. However, sensitivity analysis is a powerful tool that facilitates experts to study the stability of seismic vulnerability models. In other words, the main objective of analysing the sensitivity of the seismic vulnerability models is to find the extent to which the vulnerability degrees obtained are sensitive to the variation of input attribute values and model parameters.

This paper examines the validation of Tehran's seismic vulnerability model based on OWA operator through analysing the sensitivity of results to model parameters. OWA is a MCDM method that enables the inclusion of the optimism degree into the model. Applying the OWA operator, a spectrum of seismic vulnerability degrees are obtained regarding the decision strategy selected by experts. The main objective of this research is to examine the influence of variation of the optimism degree in the final vulnerability degrees. Minimal variability is one of the most powerful methods for deriving the order weights (Yager, 1988). The sensitivity of minimal variability OWA model of seismic vulnerability to optimism degree is analysed in this research. This analysis indicates the stability and robustness of the model. The basic contribution of this research is to propose a novel model of validating the seismic vulnerability model based on sensitivity analysis of the results. This model assumes that the North Tehran Fault is activated. Tehran's 1996 census data is used to extract the contributing attribute values. 
While it is impossible to compare the results of SLE models with the damage caused by a real earthquake, the sensitivity analysis is one of the most significant tools of validating the model. The precision of SLE models substantially depends on the uncertainty involved in the input attribute values and model parameters. Baker and Cornell (2008) examined the propagation of uncertainty in SLE models. Moreover, they proposed a model for assessing the efficiency of results provided by SLE models. In MCDM the impact of uncertainty involved in input attribute values could be analysed using Monte Carlo method (Bullard and Sebald, 1988). Emmi and Horton (1995) proposed a GISbased model applying Monte Carlo simulation for estimating the seismic loss with uncertain attribute values. Jahanpeyma et al. (2007) simulated spatial error propagation in SLE models using Monte Carlo method. They realized that there is an inverse relationship between the number of iterations required to enhance the precision of SLE model and the square root of precision (Jahanpeyma et al., 2011).

In addition to uncertain input attribute values, uncertain model parameters could also reduce the robustness of output vulnerability degrees obtained using a MCDM method (Borenstein, 1998).

Samadi Alinia and Delavar (2011) proposed an accuracy measure for Tehran's seismic vulnerability model based on granular computing which shows the average proportion of granules (urban statistical units) correctly classified based on the association rules. Khamespanah et al. (2013) improved the granular computing model of SLE by considering the exception rules as well as association rules.

A number of MCDM methods including OWA operator take the optimism degree into account. Therefore, any uncertainty in optimism degree (as a widely effective model parameter) could cause an uncertain vulnerability degree Zarghaami et al. (2007). Sensitivity analysis enables experts to find out how sensitive are the result to the optimism degree. In other words, the sensitivity analysis of OWA-based model of SLE distinguishes how much the obtained vulnerability degrees change by any variation in optimism degree (Ben-Arieh, 2005; Torra, 2001; Zarghami and Szidarovszky, 2008; Zarghami et al., 2008). Ben-Arieh (2005) proposed a method for measuring the sensitivity of the quantifier-guided OWA-based models. Zarghami et al. (2008) improved this method by developing it for minimal variability OWA operators. In the current research minimal variability OWA is implemented in order to analyse the sensitivity of OWA-based models of Tehran's seismic vulnerability assessment.

\section{METHODS AND MATERIALS}

\subsection{OWA Operator}

(Yager, 1988) introduced an umbrella group of operators that not only is able to satisfy all (And operator) and at least one (Or operator) of criteria but also it could satisfy a favourable proportion of criteria. The new aggregation operator is named ordered weighted averaging operator. OWA aggregation function is defined by Equation (1) (Yager, 1988):

$F\left(a_{1}, a_{2}, \ldots, a_{n}\right)=\sum_{j=1}^{n} w_{j} b_{j}=w_{1} b_{1}+w_{2} b_{2}+\cdots+w_{n} b_{n}$

where $\quad a_{i}=$ the attribute value associated with $i$ th criteria $b_{j}=j$ th largest value in input values

$F=$ aggregated goodness measure for an alternative

\subsection{OWA Weights}

The only parameter defining the properties of OWA operator is $W$ (the weight vector). Different weight vectors lead to different aggregation functions (Filev and Yager, 1998). The weight vector also specifies the optimism degree, which is closely related to decision strategy used by experts. Therefore, OWA operator can cover a wide range of operators from Min (low risk) to Max (high risk) operator (Filev and Yager, 1998). W = $[1,0,0, \ldots, 0]$ simulates Max operator, while $\mathrm{W}=[0,0, \ldots, 0$, 1] changes the OWA into Min operator. A Weighted Linear Combination (WLC) operator is built using $\mathrm{W}=[1 / \mathrm{n}, 1 / \mathrm{n}, \ldots$, $1 / \mathrm{n}]$ as the weight vector (Drobne and Lisec, 2009). However, for all non-equal order weights OWA turns into a non-linear aggregation operator. A number of methods have been developed to derive order weights. Some of them are based on using natural language expressions known as linguistic quantifiers(Yager, 1996). These methods tend to specify the proportion of criteria that are going to be satisfied by linking a linguistic quantifier to OWA operator. A number of methods are based on minimum variance of order weights (Fullér and Majlender, 2003). Minimizing the variance of order weights enables experts to make a decision based on all information content of attribute values.Min et al. (2007) indicated that normal distribution can be used in order to obtain OWA weights, when the input attributes are random variables. Table 1 indicates the main methods proposed to derive order weights.

\begin{tabular}{|l|l|}
\hline \multicolumn{1}{|c|}{ Method to derive order weights } & \multicolumn{1}{c|}{ Reference } \\
\hline $\begin{array}{l}\text { Maximizing the entropy of weight } \\
\text { vector }\end{array}$ & (Fullér and Majlender, \\
$\begin{array}{l}\text { Learning OWA weights from } \\
\text { observations }\end{array}$ & (Filev and Yager, 1994) \\
Using exponential function & (Xu, 2005) \\
Minimizing the variance of weight & (Fullér and Majlender, \\
vector & 2003) \\
Probabilistic OWA & (Merigó, 2009) \\
$\begin{array}{l}\text { Pairwise comparison between } \\
\text { criteria }\end{array}$ & (Boroushaki and \\
& Malczewski, 2008; \\
& Yager and Kelman, \\
Using linguistic quantifiers & 1999) \\
& (Malczewski, 2006; \\
\hline
\end{tabular}

Table 1. Fundamental methods of deriving OWA weights

\subsection{Minimal Variability OWA}

Minimal variability is one of the models proposed to derive OWA order weights (Fullér and Majlender, 2003). The main objective of minimal variability is to necessitate the OWA operator to use all information content provided by attribute values (Filev and Yager, 1998). (Yager, 1988) proposed two fundamental measures on OWA weight vector including: optimism degree and dispersion. Optimism degree is calculated using Equation (2)(Yager, 1988):

$O(W)=\frac{1}{n-1} \sum_{i=1}^{n}(n-i) * w_{i}$

where $\quad O(W)=$ calculated optimism degree of weight vector $w_{i}=$ weight associated to $i$ th largest attribute value $n=$ the number of criteria 
This measure indicates the level of optimism in a decision making process. An optimistic decision maker select the alternative which has the maximum similarity to the favourable solution, while a pessimistic decision maker tends to select the alternative with minimum similarity to the unfavourable solution (Chen, 2011). The optimism degree ranges from 0 to 1 . For a pessimistic OWA operator $\mathrm{O}(\mathrm{W})=0$, while $\mathrm{O}(\mathrm{W})=1$ indicates an optimistic OWA operator. (Yager, 1988) proved that optimism degree is not enough to describe the behavior of OWA operator. He introduced measure of dispersion presented by Equation (3) (Yager, 1988):

$D(w)=\sum_{i=1}^{n} w_{i} \ln \left(w_{i}\right)$

where $D(W)=$ dispersion of the weight vector

Larger values of dispersion ensure that different criteria have approximately the same effect on the vulnerability assessment problem. However, order weights are not equal. Therefore, the weight vector associated to an OWA operator with a predefined optimism degree is derived by solving a constraint optimization problem(Yager, 1988). Fullér and Majlender (2003) indicates that using the minimal variability constraint leads to more efficient results. In order to obtain the weight vector, they proposed the following optimization problem:

Minimize $\quad D^{2}(W)=\sum_{j=1}^{n} \frac{1}{n}\left(w_{j}-E(W)\right)^{2}=\frac{1}{n} \sum_{j=1}^{n} w_{j}^{2}-$ $\left(\frac{1}{n} \sum_{j=1}^{n} w_{j}\right)^{2}=\frac{1}{n} \sum_{j=1}^{n} w_{j}^{2}-\frac{1}{n^{2}}$

Subject to $\frac{1}{n-1} \sum_{j-1}^{n}(n-j) * w_{j}=0, \quad \sum_{i=1}^{n} w_{i}=1$.

where $\quad D=$ standard deviation of weights

$W=$ weight vector

$w_{i}=$ the weight associated to the $i$ th largest attribute

value

$n=$ the number of criteria

\section{CASE STUDY}

\subsection{Study Area}

The study area of this research is Tehran Metropolitan Area. Having more than eight million inhabitants, TMA is the most crowded city of Iran (Hashemi and Alesheikh, 2012). Seismologists argue that existence of four great faults (including North Tehran Fault, North and South Rey Faults and Mosha Fault) inside and around the city makes it extremely vulnerable in future earthquake Samadi Alinia and Delavar (2011).

\subsection{Data}

Although censuses were taken in 2006 and 2010, 1996 census data is used in this paper due to availability of 1996 data. Six contributing criteria are introduced by experts (see Table 2). Based on Iranian Civil Society (ICS) buildings built before 1966 are considered as non-standard buildings. Moreover, the percentage of weak buildings built between 1966 and 1988 is considered as another affective criterion. Height of buildings are included into the model using two categories including percentage of buildings having less than four floors and percentage of buildings having 4 floors and more. The distance of each statistical unit and their average slope are determined as important factors by experts.

\begin{tabular}{|c|l|}
\hline Layer & \multicolumn{1}{c|}{ Definition } \\
\hline Slp & \multicolumn{1}{c|}{$\begin{array}{l}\text { Average slope of each statistical unit } \\
\text { Percentage of buildings having less than 4 floors } \\
\text { in each statistical unit } \\
\text { Percentage of buildings having 4 floors and more } \\
\text { in each statistical unit } \\
\text { Percentage of buildings built before 1966 in } \\
\text { each statistical unit } \\
\text { Percentage of weak buildings built between } \\
\text { Bf_666-1988 in each statistical unit } \\
\text { The geometric distance of statistical units to } \\
\text { NTF (in km) }\end{array}$} \\
Dst_F6-88
\end{tabular}

Table 2. Contributing criteria and their definitions

\subsection{Data Preparation}

The attribute values of five randomly selected statistical units are illustrated in Table 3.

\begin{tabular}{|l|l|l|l|l|l|l|}
\hline id & Slp & Ls_4 & Mr_4 & Bf_66 & Bt66-88 & Dst_F(km) \\
\hline 1 & 14 & 17 & 8 & 52 & 13 & 6.751 \\
2 & 1 & 4 & 0 & 84 & 6 & 3.890 \\
3 & 26 & 2 & 0 & 91 & 19 & 1.435 \\
4 & 4 & 88 & 0 & 97 & 0 & 5.476 \\
5 & 6 & 9 & 1 & 63 & 5 & 2.054 \\
\hline
\end{tabular}

Table 3. Attribute values of five randomly selected statistical units

Attribute values of different criteria are not in the same scale. Therefore, they cannot be aggregated directly. Attribute values are first normalized. (Malczewski, 2006) introduced a method to normalize attribute values. He classified all contributing criteria into two classes including cost and benefit criteria. Benefit criteria are a class of criteria that their larger values are favourable for the decision maker. However, lower values of cost criteria are desirable. Through the normalization process all attribute values are divided by their maximum value. Then, the attribute values of the cost criteria are subtracted bye one. In the seismic vulnerability assessment distance to the North Tehran Fault is specified as a cost criterion, while five other criteria are benefit criteria. Table 4 demonstrates the normalized attribute values of five selected statistical units.

\begin{tabular}{|l|l|l|l|l|l|l|}
\hline id & Slp & Ls_4 & Mr_4 & Bf_66 & Bt66-88 & Dst_F(km) \\
\hline 1 & 0.53 & 0.17 & 0.22 & 0.53 & 0.54 & 0.71 \\
2 & 0.03 & 0.04 & 0 & 0.86 & 0.25 & 0.84 \\
3 & 1 & 0.02 & 0 & 0.93 & 0.79 & 0.94 \\
4 & 0.15 & 0.91 & 0 & 1 & 0 & 0.77 \\
5 & 0.25 & 0.09 & 0.02 & 0.64 & 0.20 & 0.92 \\
\hline
\end{tabular}

Table 4. Normalized attribute values of five randomly selected statistical units

\subsection{Sensitivity Analysis for Minimal Variability OWA}

Figure 1 illustrates the steps undertaken in order to calculate the sensitivity of seismic vulnerability degree to optimism degree using the minimal variability model. First, the criteria that substantially affect the seismic vulnerability are detected. Then, 
these criteria are categorized into two major types including benefit and cost. The attribute values then are normalized regarding the type of the criteria. Next, experts are asked to determine the decision strategy (optimism degree). Order weights are calculated using Equation (5). Then, the sensitivity of seismic vulnerability degree is obtained for each statistical unit applying Equation (6). Sensitivity degrees are classified into five classes and the result is illustrated using ArcGIS software.

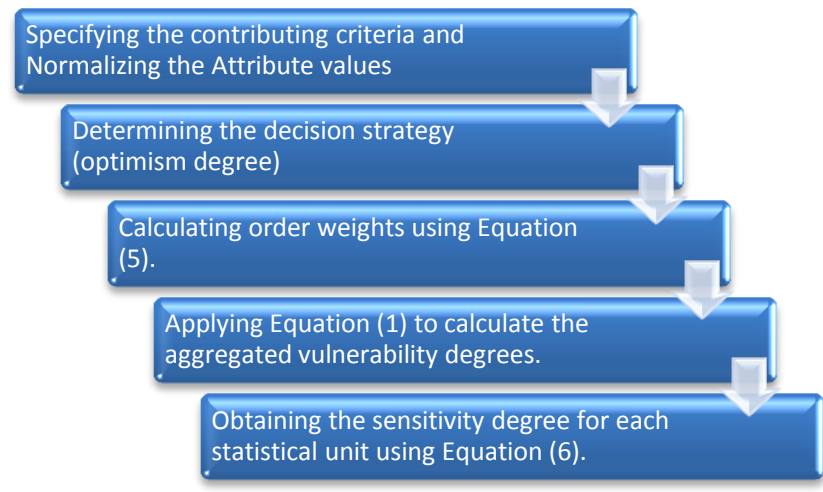

Figure 1. Steps to analyze the sensitivity of model

The order weights in minimal variability OWA is derived using Equation (5)(Zarghami et al., 2008):

$$
\begin{aligned}
w_{1} & =\frac{2(2 n-1)-6(n-1)(1-O)}{n(n+1)} \\
w_{n} & =\frac{6(n-1)(1-O)-2(n-2)}{n(n+1)} \\
w_{j} & =\frac{n-j}{n-1} w_{1}+\frac{j-1}{n-1} w_{n} j \in\{2, \ldots n-1\}
\end{aligned}
$$

where

$$
\begin{aligned}
& O=\text { optimism degree } \\
& n=\text { the number of criteria }
\end{aligned}
$$

In the proposed model of seismic vulnerability assessment there are six different criteria. Consequently, for each given optimism degree a set of six order weights is calculated which has the minimum variance among all possible weight vectors. Table 5 demonstrates the order weights for five different optimism degrees, calculated using Equation (5).

\begin{tabular}{|c|c|c|}
\hline $\mathrm{O}(\mathrm{W})$ & $\mathrm{W} 1$ & Variance \\
\hline 0 & {$[0,0.04,0.09,0.23,0.38,0.52]$} & 0.04256 \\
0.25 & {$[0,0.05,0.13,0.2,0.27,0.34]$} & 0.01691 \\
0.50 & {$[0.16,0.16,0.16,0.16,0.16,0.16]$} & 0 \\
0.75 & {$[0.34,0.27,0.2,0.13,0.05,0]$} & 0.01691 \\
1 & {$[0.52,0.38,0.23,0.09,0.04,0]$} & 0.04256 \\
\hline
\end{tabular}

Table 5. Weight vector and variance for five different optimism degree

Table 5 illustrates that lower optimism degrees $(O(W)=0)$ tend to generate larger weights at the end of weight vector, while in more optimistic decision strategies $(O(W)=1)$ larger weights are derived at the beginning of the weight vector. OWA operator sorts the attribute values in a descending order. Therefore, for $O(W)=0$, the largest attribute value among six affecting criteria is multiplied by 0 and the lowest attribute 4 value is multiplied by 0.52 . In other words, based on minimal variability OWA model of seismic vulnerability in pessimistic decision strategies, the criteria that has the minimum attribute value extremely affect the final vulnerability and for larger values of optimism degree the criteria with maximum attribute value has a considerably high effect in aggregated seismic vulnerability degree.

After deriving the weight vectors, the aggregated vulnerability degrees are calculated using Equation (1). Five distinct solutions are obtained by applying this model. In other words, for each statistical unit five different seismic vulnerability degrees are calculated. Higher optimism degree results in the higher aggregated seismic vulnerability degree. Aggregated vulnerability degrees for five selected statistical units are illustrated in Figure 2.

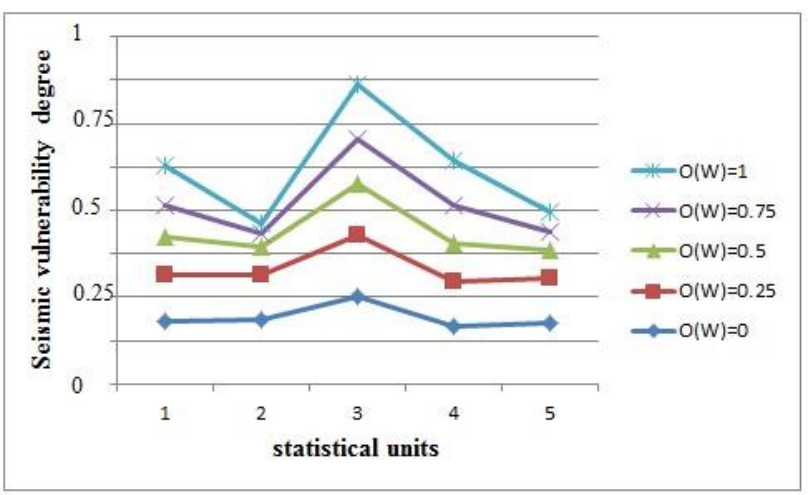

Figure 2. Aggregated seismic vulnerability degree for five randomly selected statistical units

It could be concluded from Figure 2 that optimism degree has a direct relationship with aggregated seismic vulnerability degree for $O(W)=1$ the highest values are obtained, while $O(W)=0$ lead to approximately lowest value for all five statistical units.

3.4.1 Measuring the Sensitivity of the Minimal Variability OWA Model: A wide range of seismic vulnerability degrees could be obtained using minimal variability OWA model. Sensitivity analysis of the model reveals that which one of these results is more robust and which one is more sensitive to optimism degree. Zarghami et al. (2008) calculated the sensitivity of aggregated value of minimal variability OWA using Equation (6):

$S=\frac{\partial F}{\partial O}=\frac{6}{n(n+1)} \sum_{j=1}^{\left[\frac{n+1}{2}\right]}(n-2 j+1) *\left(b_{j}-b_{n+1-j}\right)$

where $S=$ sensitivity of aggregated value to optimism degree $b_{j}=$ jth largest attribute value $n=$ the number of criteria

This measure of sensitivity indicates the extent to which the aggregated value of each statistical unit changes by variation of decision strategy (optimism degree)(Zarghaami et al., 2007). In minimal variability OWA based model of seismic vulnerability assessment, the aggregated value denotes the vulnerability degree. Therefore, the sensitivity measure introduced in Equation (6) demonstrates the extent to which the vulnerability degree of a statistical unit is sensitive to the variation of optimism degree. The less sensitive the vulnerability degree of a 
statistical unit, the more robust the vulnerability degree is calculated under the uncertainty of optimism degree. Figure 3 demonstrates aggregated seismic vulnerability degrees and their calculated sensitivity for the five selected statistical units. The results indicate that the sensitivity degree has a direct relationship with vulnerability degree. In other words, the vulnerability degree of vulnerable and very vulnerable statistical units is more sensitive to optimism degree in comparison to lower seismic vulnerable statistical units.

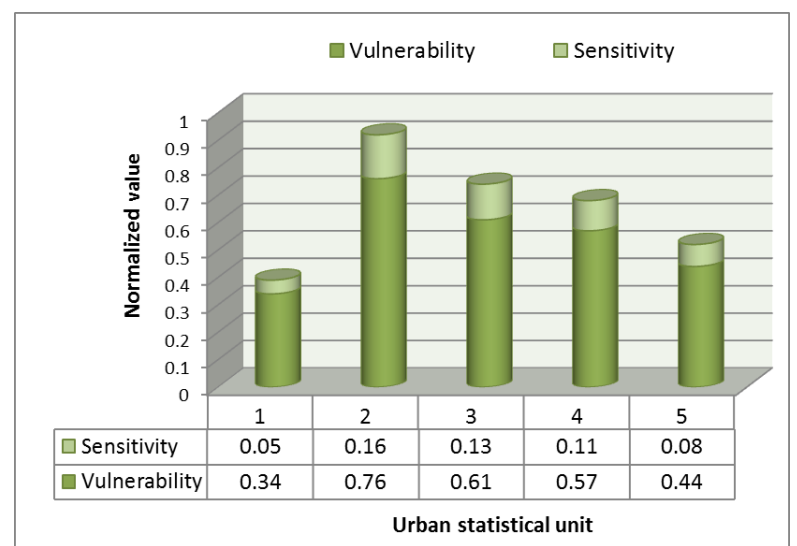

Figure 3. Sensitivity and vulnerability measures for five randomly selected statistical units

Figure 4 demonstrates the sensitivity degree for all statistical units in TMA. A number of statistical units in southern part of Tehran and most of the units in northern part of the city are very sensitive to optimism degree. It means that uncertainty involved in optimism degree results in high uncertainty in seismic vulnerability of these statistical units.

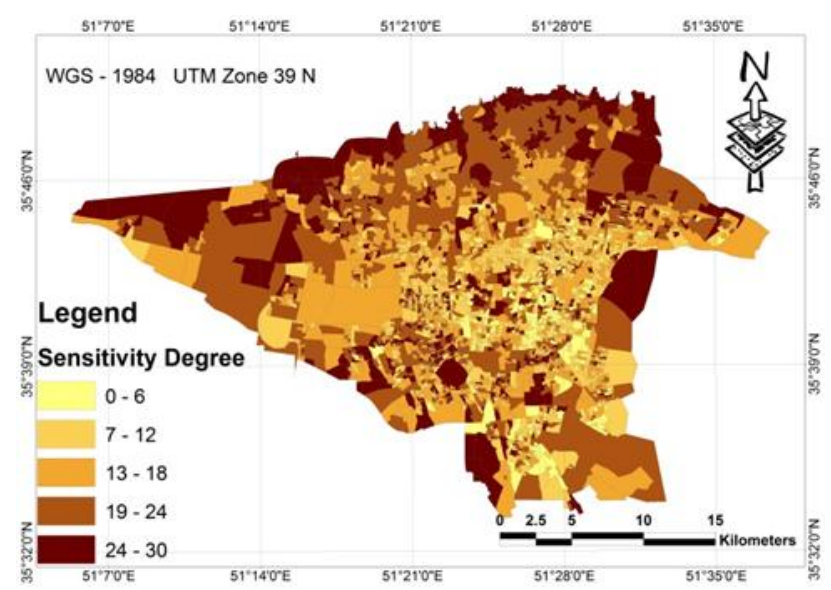

Figure 4. Sensitivity measure for statistical units of Tehran Metropolitan Area

A number of statistical units in northern part of Tehran are vulnerable and very vulnerable due to the very short distance to North Tehran Fault. Non-standard construction causes a number of statistical units to be very vulnerable. Due to direct relationship between vulnerability degree and sensitivity of vulnerability degree, the statistical units which are vulnerable are very sensitive as well.

\section{DISCUSSION}

In ill-structured multi-criteria decision making problems such as seismic vulnerability assessment and loss estimation, in order to compare the efficiency of models, additional measures are required. One of the most significant measures that facilitate the comparison of different models is sensitivity measure. Calculating the sensitivity of vulnerability degrees of statistical units beside their vulnerability degrees enables experts to compare the stability and robustness of models. In minimal variability OWA model of seismic vulnerability assessment, the maximum of sensitivity measure is 0.27 which is $25 \%$ of its vulnerability degree. In other words, the variation of optimism degree will make a fluctuation between $0.9 \%$ and $25 \%$ in aggregated vulnerability degree. It means that the model considerably depend on optimism degree. However, in a number of safer areas, the model has an acceptable robust behaviour. In other words, in the minimal variability OWA, a more stable decision is made for the alternatives which have a lower aggregated value (safer statistical unit). As a conclusion, the minimal variability OWA model of seismic vulnerability assessment has a robust behaviour under uncertainty of optimism degree if the statistical units are not vulnerable or very vulnerable.

\section{CONCLUSION}

This paper proposed a model to analyse the sensitivity of seismic vulnerability degrees obtained by using minimal variability OWA operator. This additional measure facilitates seismic loss estimation under uncertainty. Sensitivity measure of seismic vulnerability model demonstrates which statistical unit have a more stable seismic vulnerability degree. The research indicates that sensitivity of seismic vulnerability degrees has a direct relationship with seismic vulnerability degrees. Consequently, the seismic vulnerability degree obtained from minimal variability OWA is more robust for safer statistical units, while for vulnerable and very vulnerable statistical units a higher level of uncertainty is involved in aggregated vulnerability degree. The results also demonstrate that the minimal variability OWA model for seismic vulnerability assessment have more precise values in central areas of TMA especially in areas that are not vulnerable to earthquake. However, the minimal variability OWA model is noticeably sensitive to optimism degree in northern areas and a number of southern parts which are more vulnerable to earthquake. Although this paper proposed a model to analyse the internal sensitivity of minimal variability OWA model to some extents, further researches may be focused on external accuracy of the model which could be calculated either by comparison of results with real earthquake damages (if happens in future) or by comparison to results obtained using other models of seismic vulnerability assessment.

\section{REFERENCES}

Samadi Alinia, H. S., and Delavar, M., 2011. Tehran's seismic vulnerability classification using granular computing approach. Applied Geomatics, 3(4), 229-240.

Baker, J. W., and Cornell, C. A., 2008. Uncertainty propagation in probabilistic seismic loss estimation. Structural Safety, 30(3), pp. 236-252. 
Ben-Arieh, D., 2005. Sensitivity of multi-criteria decision making to linguistic quantifiers and aggregation means. Computers and Industrial Engineering, 48(2), pp. 289-309.

Borenstein, D., 1998. Towards a practical method to validate decision support systems. Decision Support Systems, 23(3), pp. 227-239.

Boroushaki, S., and Malczewski, J., 2008. Implementing an extension of the analytical hierarchy process using ordered weighted averaging operators with fuzzy quantifiers in ArcGIS. Computers and Geosciences, 34(4), pp. 399-410.

Bullard, C. W., and Sebald, A. V., 1988. Monte Carlo sensitivity analysis of input-output models. The Review of Economics and Statistics, pp. 708-712.

Chen, T.-Y., 2011. Optimistic and pessimistic decision making with dissonance reduction using interval-valued fuzzy sets. Information Sciences, 181(3), pp. 479-502.

Crowley, H., and Bommer, J. J., 2006. Modelling seismic hazard in earthquake loss models with spatially distributed exposure. Bulletin of Earthquake Engineering, 4(3), pp. 249-273.

Drobne, S., and Lisec, A., 2009. Multi-attribute decision analysis in GIS: weighted linear combination and ordered weighted averaging. nature, 4(26), 28.

Emmi, P. C., and Horton, C. A., 1995. A Monte Carlo simulation of error propagation in a GIS-based assessment of seismic risk. International Journal of Geographical Information Systems, 9(4), pp. 447-461.

Filev, D., and Yager, R. R., 1994. Learning OWA operator weights from data. In the Proceedings of the Third IEEE Conference on the Fuzzy Systems, 1994. IEEE World Congress on Computational Intelligence.

Filev, D., and Yager, R. R., 1998. On the issue of obtaining OWA operator weights. Fuzzy Sets and Systems, 94(2), pp. 157-169.

Fullér, R., and Majlender, P., 2001. An analytic approach for obtaining maximal entropy OWA operator weights. Fuzzy Sets and Systems, 124(1), pp. 53-57.

Fullér, R., and Majlender, P. 2003. On obtaining minimal variability OWA operator weights. Fuzzy Sets and Systems, 136(2), pp. 203-215.

Hashemi, M., and Alesheikh, A., 2012. Development and implementation of a GIS-based tool for spatial modeling of seismic vulnerability of Tehran. Natural Hazards and Earth System Science, 12(12), pp. 36593670 .

Jahanpeyma, M., Delavar, M., Malek, M., and Kamalian, N., 2007. Analytical evaluation of uncertainty propagation in seismic vulnerability assessment of Tehran using GIS.In the Proceedings of the International Symposium of Spatial Data Quality(ISSDQ 2007).The netherland

Khamespanah, F., Delavar, M. R., Alinia, H. S., and Zare, M., 2013. Granular Computing and Dempster-Shafer Integration in Seismic Vulnerability Assessment Intelligent Systems for Crisis Management pp. 147158.

Kolat, Ç., Doyuran, V., Ayday, C., and Lütfi Süzen, M. 2006. Preparation of a geotechnical microzonation model using Geographical Information Systems based on Multicriteria Decision Analysis. Engineering geology, 87(3), pp. 241-255.

Malczewski, J., 2006. Ordered weighted averaging with fuzzy quantifiers: GIS-based multicriteria evaluation for land-use suitability analysis. International Journal of
Applied Earth Observation and Geoinformation, 8(4), pp. $270-277$.

Merigó, J. M., 2009. Probabilistic Decision Making with the OWA Operator and its Application in Investment Management. In proceedings of the IFSA/EUSFLAT Conf.

Min, D., Xu-rui, Z., and Yun-xiang, C., 2007. A Note on OWA Operator Based on the Normal Distribution. In proceedings of the Management Science and Engineering, 2007. ICMSE 2007. International Conference on.

Mohanty, W. K., Walling, M. Y., Nath, S. K., and Pal, I., 2007. First order seismic microzonation of Delhi, India using geographic information system (GIS). Natural Hazards, 40(2), pp. 245-260.

Rashed, T., and Weeks, J., 2003. Assessing vulnerability to earthquake hazards through spatial multicriteria analysis of urban areas. International Journal of Geographical Information Science, 17(6), pp. 547576.

Torra, V., 2001. Sensitivity analysis for WOWA, OWA and WM operators. Paper presented at the Industrial Electronics, 2001. In proceedings of the IEEE International Symposium on ISIE 2001.

$\mathrm{Xu}, \mathrm{Z}$., 2005. An overview of methods for determining OWA weights. International Journal of Intelligent Systems, 20(8), pp. 843-865.

Yager, R. R., 1988. On ordered weighted averaging aggregation operators in multicriteria decisionmaking. Systems, Man and Cybernetics, IEEE Transactions on, 18(1), pp. 183-190.

Yager, R. R., 1996. Quantifier guided aggregation using OWA operators. International Journal of Intelligent Systems, 11(1), pp. 49-73.

Yager, R. R., and Kelman, A., 1999. An extension of the analytical hierarchy process using OWA operators. Journal of Intelligent and fuzzy Systems, 7(4), pp. 401-417.

Zarghaami, M., Ardakanian, R., and Szidarovszky, F., 2007. Obtaining robust decisions under uncertainty by sensitivity analysis on OWA operator. In the proceedings of the Computational Intelligence in Multicriteria Decision Making, IEEE Symposium on.

Zarghami, M., and Szidarovszky, F., 2008. Fuzzy quantifiers in sensitivity analysis of OWA operator. Computers and Industrial Engineering, 54(4), pp. 1006-1018.

Zarghami, M., Szidarovszky, F., and Ardakanian, R., 2008. Sensitivity analysis of the OWA operator. Systems, Man, and Cybernetics, Part B: Cybernetics, IEEE Transactions on, 38(2), pp. 547-552. 